Meta

Journal des tradlucteurs

Translators' Journal

\title{
La traduction des formes verbales du présent français en bafia
}

\section{Dieudonné P. Aroga Bessong}

Volume 27, numéro 3, septembre 1982

URI : https://id.erudit.org/iderudit/002068ar

DOI : https://doi.org/10.7202/002068ar

Aller au sommaire du numéro

Éditeur(s)

Les Presses de l'Université de Montréal

ISSN

0026-0452 (imprimé)

1492-1421 (numérique)

Découvrir la revue

Citer cet article

Aroga Bessong, D. P. (1982). La traduction des formes verbales du présent français en bafia. Meta, 27(3), 257-270. https://doi.org/10.7202/002068ar d'utilisation que vous pouvez consulter en ligne. 


\title{
LA TRADUCTION DES FORMES VERBALES DU PRÉSENT FRANÇAIS EN BAFIA*
}

\author{
Dieudonné P. Aroga Bessong
}

Un des problèmes que doit résoudre le traducteur du français en bafia est la traduction des catégories morphologiques verbales françaises. Dans les pages qui suivent, nous nous limitons au mode indicatif et dans ce cadre, à un des grammèmes de la catégorie du temps : le présent.

En français, le terme «présent» n'est qu'une simple étiquette qui trouve sa justification dans la description morphologique du système verbal de cette langue, mais qui du point de vue sémantique est insatisfaisant.

En effet, le présent français est polysémique. À l'indicatif, il caractérise l'événement grosso modo comme coïncidant avec le moment du discours. Mais en plus, il comporte plusieurs autres significations ou valeurs sémantiques, dont les principales sont le présent actuel, le présent historique, le présent gnomique, et le présent habituel (cf. 1. 2.1-4 pour les exemples).

La traduction en bafia du présent français dans ses différentes valeurs exige du traducteur deux types de choix logiques non ordonnés: 1 . Le premier type porte sur trois catégories verbales bafias (en plus du mode indicatif): le temps (choisi surtout entre le présent et le quasi-passé), l'absolutivité (choisie entre la forme absolue et la forme relative), et l'aspect (choisi entre le ponctuel, le duratif, l'habituel et l'itératif). 2 . Le deuxième type porte sur les différentes significations ou valeurs sémantiques du présent et du quasi-passé bafia, et de chacun des grammèmes de l'absolutivité et de l'aspect retenus. À ce niveau, le traducteur doit se soumettre aux contraintes spécifiques de la combinatoire des valeurs sémantiques des aspects du bafia avec celles des grammèmes du temps impliqués.

Le problème initial de la traduction des catégories morphologiques verbales, et plus précisément de leurs grammèmes, est donc en réalité celui de l'identification des valeurs sémantiques de ces derniers dans les deux langues et de l'établissement de correspondances entre les différentes valeurs identifiées.

* Le présent article est une partie d'un mémoire de maittrise en traduction de la section traduction du département de linguistique de l'Université de Montréal.

L'auteur a bénéficié d'une bourse d'études offerte par l'Agence canadienne de développement international, et obtenue par l'entremise du gouvernement de la république unie du Cameroun, durant toute la période d'études pour mériter le grade sus-mentionné. Il lui est agréable d'exprimer ici sa gratitude aux dirigeants des institutions en question.

Il tient également à signifier sa profonde reconnaissance à Igor A. Mel'čuk, Lidija N. Iordanskaja, et André Clas qui ont accepté de lire une ou plusieurs des versions préparatoires du présent article. Leurs conseils, critiques et suggestions ont permis d'en améliorer la présentation. Toutes les erreurs qui ont pu subsister ne sont imputables qu'à l'auteur. 
Le but du présent article est de proposer une solution à ce problème, dans le cadre de la théorie linguistique «Sens $\Leftrightarrow$ Texte» (MST) (Mel'čuk, 1978). Cette solution sera présentée sous la forme d'un modèle de traduction, à savoir un ensemble de règles faisant correspondre au sens français concerné, le ou les sens bafia(s) correspondant(s).

Tout comme E. A. Nida, J.-P. Vinay et beaucoup d'autres, les théoriciens du MST envisagent la traduction comme une opération complexe comportant trois étapes majeures : 1. Adoptant la perspective analytique, le traducteur détermine le sens du texte de départ en y appliquant les règles de la langue en question. II procède donc du texte vers le sens : c'est l'analyse ou décodage. 2 . Le traducteur transforme ensuite le sens de départ ainsi obtenu pour lui permettre d'être exprimé dans le texte d'arrivée. Cette étape implique dans une grande mesure le domaine extra-linguistique. Elle peut être exclue lorsque les circonstances l'indiquent, en d'autres termes lorsque la correspondance des sens dans les langues en question ne pose aucun problème. Il convient de souligner le rôle déterminant de la langue d'arrivée à ce niveau. En effet, l'aménagement du sens de départ n'est envisagé que dans le but de le rendre exprimable dans la langue d'arrivée, selon les exigences de cette dernière. 3. Adoptant la perspective synthétique, le traducteur construit le texte d'arrivée en appliquant au sens transformé (selon le cas), les règles de la langue d'arrivée. Il procède donc du sens vers le texte : c'est la synthèse ou encodage.

Le modèle de traduction des formes verbales françaises en bafia que nous proposons rend compte uniquement de la seconde des trois étapes de la traduction. Ledit modèle sera un ensemble de règles établissant les correspondances entre les différentes valeurs sémantiques du présent français, et chacune des valeurs du présent ou du quasi-passé bafia, muni des valeurs des grammèmes de l'absolutivité et de l'aspect autorisées par la combinatoire des valeurs mentionnées plus haut.

Il faut se garder de penser qu'il en résulte une traduction exacte au sens de Wierzbicka (Mel'čuk, 1978: 295), puisque les catégories ou grammèmes impliquées dans la présente étude ne jouent pas le même rôle morphologique en français qu'en bafia (déjà, le bafia en présente un plus grand nombre!). S'il en était ainsi, l'étape dont nous entendons rendre compte serait (en partie) inutile, puisque les correspondances entre les deux langues seraient exactes, et qu'alors tout autre phénomène nécessaire pour une bonne compréhension du texte de départ relèverait du domaine extra-linguistique, et donc se trouverait en dehors du donné proprement linguistique dont le MST comme tel ordonne la production.

Le présent de l'indicatif français peut être rendu en bafia par les catégories morphologiques verbales suivantes: le temps (dont seuls sont impliqués le présent, le quasi-passé), l'aspect (ponctuel, duratif, habituel, itératif), l'absolutivité (absolutif, relatif). Dans les pages qui suivent, seuls seront considérés le présent de l'indicatif français, et les catégories sus-mentionnées du verbe bafia.

Nous ne tiendrons donc pas compte des autres catégories morphologiques, soit la personne et le nombre dans les deux langues, auxquels il convient d'ajouter le genre pour le français et la classe pour le bafia: leurs significations 
peuvent être assez facilement reliées par correspondance, d'une langue à l'autre, selon un schéma clair et net.

Nous négligerons également le choix des lexèmes correspondants pour une forme verbale donnée.

Ainsi, étant donné les phrases suivantes :

(1) a. Il coupe du bois maintenant.

b. á-réndèn fínén bi čàmèn kí

3,I-PRES, ABS-couper-DUR bois dans moment celui-ci

Les règles du modèle que nous proposons ne rendront compte, ni de la correspondance entre les deux lexèmes verbaux (français = COUPER; bafia = RìRÉN), ni de la personne, du nombre, de la classe ou le genre (selon le cas), dans les deux formes verbales, mais plutôt de la correspondance entre le présent français d'une part, et le présent duratif absolu bafia de l'autre.

La résolution du problème ainsi délimité est effectuée en quatre étapes:

I - Analyse du présent français :

1. Description générale.

2. Liste des valeurs sémantiques.

Les données recueillies dans cette étape sont tirées d'une comparaison de l'analyse sémantique du présent français dans Chevalier et al. (1964), Grévisse (1980), Martinet et al. (1979), Schogt (1968), et Wagner et Pinchon (1962). Elles sont donc tenues pour acquises.

II - Présentation des significations morphologiques du bafia retenues :

1. Description générale

2. Liste des valeurs sémantiques des grammèmes

3. Combinatoire de valeurs sémantiques des grammèmes.

Les données présentées dans cette étape sont tirées d'une description de la conjugaison des verbes bafias à l'indicatif: Aroga et Mel'čuk (1981), en ce qui concerne la première partie de cette étape, et de l'intuition linguistique de l'auteur du présent article, en ce qui concerne les deux autres.

III - Commentaires généraux sur les correspondances entre les catégories analysées des deux langues.

IV - Règles de correspondance sémantique entre les valeurs du présent français, et celles des absolutivités, aspects et temps bafia correspondants.

\section{I - LE PRÉSENT FRANÇAIS}

\section{I.1. Description générale}

Le présent français caractérise l'événement décrit par le verbe comme incluant le moment du discours sur le plan temporel. Chevalier et al. (1964: 336) déclare: "Le présent est constitué du passé qui vient de s'écouler et du futur qui s'amorce»; abondant dans le même sens, Wagner et Pinchon (1962: 336) y voient «la forme au moyen de laquelle le locuteur ou le narrateur exprime tout ce qui constitue son actualité, tout ce qui s'y rattache.» Mais, comme le 
souligne Grévisse (1980: 831) le présent au sens strict décrit «un fait qui a lieu au moment où l'on parle.»

\subsection{Liste des valeurs du présent français}

Dans le tableau 1 , les différentes valeurs sémantiques reconnues du présent sont réparties en fonction de la classification de Chevalier et al. pour faire ressortir les ressemblances. La terminologie des auteurs a été, autant que possible, préservée. Les principales valeurs sémantiques indiquées sont reprises et décrites par la suite.

\section{TABLEAU 1}

Valeurs sémantiques reconnues du présent français

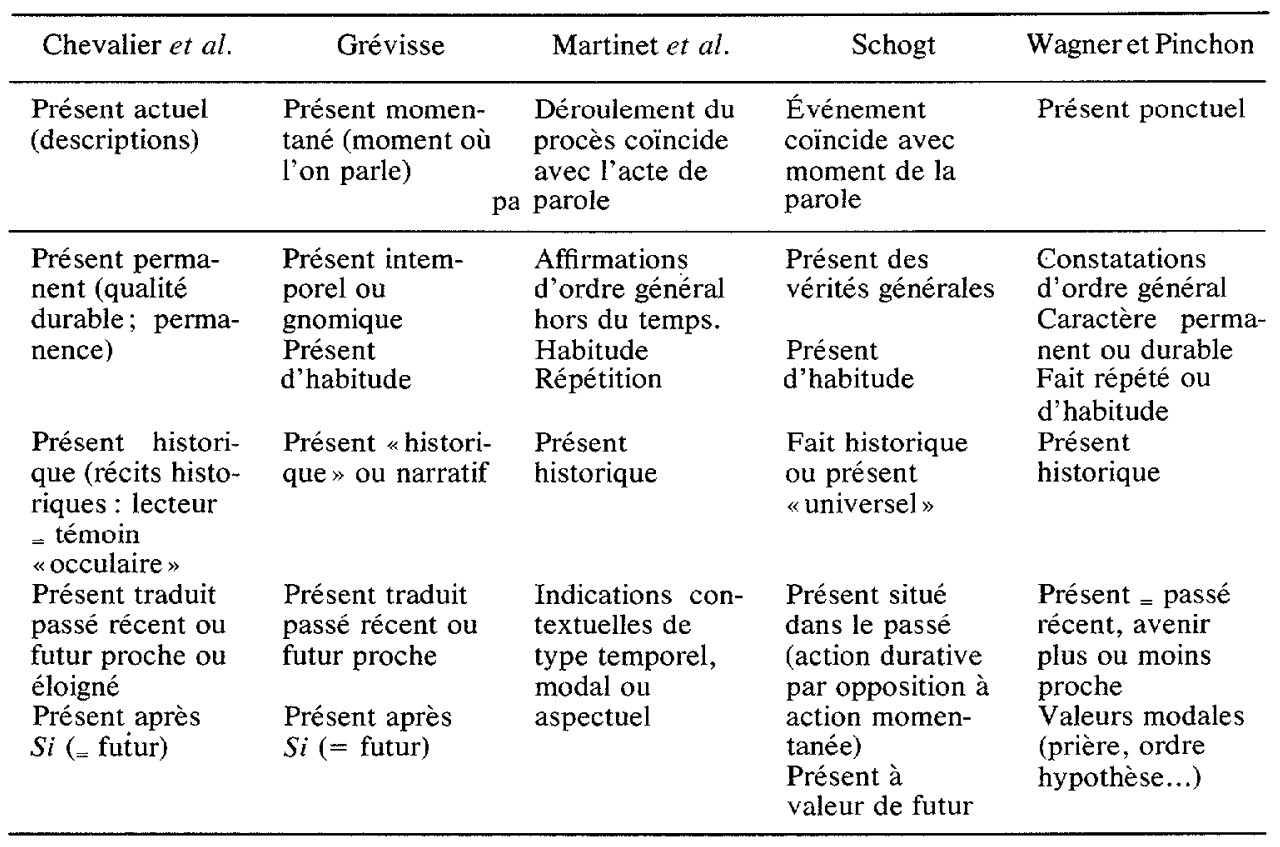

L'observation du tableau ci-dessus permet de tirer deux conclusions:

1. le présent comporte trois principaux types de valeurs sémantiques: a) les valeurs temporelles; b) les valeurs aspectuelles; c) les valeurs modales.

Nous ne tiendrons pas compte des valeurs modales parce qu'elles nous semblent moins représentatives du présent. Nous ne traitons donc que des valeurs non modales (cf. a et b).

2. En tenant compte des valeurs non modales du présent, il se dégage une impression d'homogénéité par-delà les différences d'analyse ou de dénomination des valeurs identifiées. Toutes les valeurs concernées se groupent en deux classes distinctes, selon que le présent en question se substitue ou non à un autre temps de la conjugaison française. Les valeurs «intrinsèques» du 
présent seront celles qui ne constituent pas (de façon primordiale) une substitution temporelle, les autres étant dites «contingentes».

Les valeurs intrinsèques du présent français sont selon nous au nombre de quatre. Elles peuvent être groupées deux à deux comme le montre le tableau 2.

TABLEAU 2

Structure des valeurs intrinsèques du présent français

\begin{tabular}{|c|c|c|}
\hline $\mathbf{N}^{\circ}$ & Valeur & Regroupement \\
\hline 1 & Présent actuel & \multirow{2}{*}{ actuel } \\
\hline 2 & Présent historique & \\
\hline 3 & Présent gnomique & \multirow{2}{*}{ usuel } \\
\hline 4 & Présent habituel & \\
\hline
\end{tabular}

Les valeurs temporelles contingentes du présent français sont moins systématiques et incluent : - le présent comme expression d'un passé récent; - le présent comme expression d'un futur plus ou moins éloigné ${ }^{1}$.

Il est nécessaire de reprendre chaque valeur intrinsèque du présent français pour en préciser les conditions d'apparition. Celles des valeurs contingentes sont données globalement.

1.2.1. Le présent actuel [PRESF.(ACT)]. Il apparaît lorsque la forme verbale décrit un événement qui advient au même moment que l'acte de parole. En cela, il s'apparente à la forme progressive de l'anglais, comme le montre l'exemple ci-dessous.

Aroga rend visite à son ami Zock et trouve ce dernier écrivant une lettre. Aroga qui vient juste d'entrer, demande à Zock ce qu'il est en train de faire et Zock lui répond :

(2) J'écris une lettre à Assen ( $\mathrm{I}$ am writing a letter to Assen).

I.2.2. Le présent historique [PRES F(HIST)]. Il apparaît lorsque la forme verbale décrit un événement du temps révolu, inclus dans un énoncé narratif, qu'il s'agisse d'un récit, d'un compte rendu, ou d'un reportage. Il a pour rôle de permettre à l'auditeur (lecteur) d'être en quelque sorte témoin d'un événement (ou une suite d'événements) situé dans le temps révolu, mais présenté comme faisant partie de l'actualité (Wagner et Pinchon 1962: 338) de l'auditeur. En effet, le contexte temporel est clairement fixé au préalable dans le temps révolu par une marque précise (une forme verbale au passé, un adverbe de temps, etc.)

Ainsi, Aroga fait le récit du règne de Baban, le défunt chef de son village natal. Au début du récit, le temps a été clairement fixé comme étant révolu. Aroga en arrive à la mort du chef et il énonce la phrase (3) :

1. L'emploi du présent après si est considéré dans notre classification comme modal, donc exclu de nos préoccupations. 
(3) Baban le chef des Moukos meurt après l'indépendance.

Un autre exemple serait la phrase (4):

(4) Hier, alors que je me promenais dans le bois, je vois un serpent.

Remarques: 1) Le contexte historique dans lequel advient l'événement décrit par la forme verbale au présent historique peut être connu d'avance par l'auditeur, ou supposé tel (comme le serait la mort du chef Baban pour les Moukos). L'interprétation sémantique du présent français comme historique le rapproche alors de celle du même présent comme gnomique ou intemporel dans la même langue (cf. I.2.3 ci-dessous).

2) Le présent historique français peut apparaitre immédiatement après une marque temporelle non verbale déterminant comme il se doit le contexte temporel (révolu) indispensable à l'apparition dudit présent. Selon Schogt (1968: 31), un tel présent indique une action durative et s'oppose au second présent de la phrase (cf. (4)), décrivant quant à lui une action ponctuelle, comme en (5).

(5) Hier, je sors de la maison et je vois un serpent.

L'emploi du présent dans la première forme verbale d'une telle phrase commande celui de la seconde (mais pas inversement).

I.2.3. Le présent gnomique. [PRES $\mathrm{F}(\mathrm{GNOM})]$. Il apparaît lorsque l'événement décrit par la forme verbale constitue l'énonciation d'une vérité d'ordre général, permanent ou du moins durable. Il est utilisé dans les proverbes, les aphorismes, les descriptions de phénomènes naturels (par exemple la caractérisation des mœurs animales ou humaines), les exposés scientifiques ou techniques (par exemple la description d'une machine).

(6) a. La terre tourne autour du soleil.

b. Les Pharisiens et tous les autres juifs obéissent à l'enseignement de leurs ancêtres.

c. Les abeilles communiquent au moyen de la danse en huit.

d. Une voiture a quatre roues.

I.2.4. Le présent habituel. [PRES $\mathrm{F(HAB)}$ ]. Il apparaît lorsque la forme verbale en cause décrit un événement habituel. Le contexte dans lequel elle est manifestée facilite généralement son interprétation. Les éléments contextuels qui fournissent l'information nécessaire sont souvent des compléments circonstanciels temporels connotant l'habitude ou la répétition imprécise.

(7) a. Mon père écoute le bulletin des nouvelles le matin.

b. Mon père écoute le bulletin des nouvelles tous les matins.

I.2.5. Les valeurs contingentes. Elles sont intimement liées aux différents contextes où le présent peut être interprété comme un passé récent ou un futur proche. Des compléments ou des propositions subordonnées temporelles (ou conditionnelles pour les emplois conditionnels du présent à valeur de futur) apportent alors l'information requise.

(8) a. Je quitte à l'instant mon ami.

b. Hier, je m'embourbe en faisant marche arrière avec ma voiture.

c. Mon père arrive demain à Paris.

d. Le mariage a lieu dans trois jours. 


\section{II - LES CATÉGORIES MORPHOLOGIQUES DU BAFIA RETENUES}

Les catégories morphologiques verbales bafias retenues sont, rappelons-le : - l'abolutivité ; - l'aspect ; - le temps, dont nous ne considérons que le présent et le quasi-passé. Elles seront étudiées dans cet ordre.

\section{II.l. Description générale}

II.1.1. L'absolutivité. La catégorie morphologique verbale bafia d'absolutivité permet de caractériser une forme verbale donnée comme étant ou non la tête d'un groupe de formes verbales coordonnées. Elle joue donc un rôle purement syntaxique.

L'absolutivité du bafia comporte deux grammèmes :

a) L'absolutif [ABS]. Il identifie la forme verbale qui le manifeste comme la tête d'un groupe de formes verbales coordonnées, ou l'unique forme verbale d'une phrase.

b) Le relatif $[\mathrm{REL}]$. Il identifie la forme verbale qui le manifeste comme membre d'un groupe de formes verbales coordonnées dont elle n'est pas la tête; celle-ci est, au reste, dûment mise en évidence. Le verbe au relatif n'est donc jamais manifesté seul (en principe) dans une phrase. Son temps correspond à celui de la tête du groupe coordinatif.

Il convient de souligner que l'absolutif et le relatif sont respectivement marqués dans les formes verbales du bafia par la présence, ou l'absence d'une expression claire du temps.

Les exemples suivants illustrent la description de l'absolutivité ainsi esquissée.
(9) a. Àrógà á-kànkàn 3,I-PRES, ABS-écrire, DUR 3,I-REL-écouter, DUR
fjém.
fjém.
fjém.

Remarque: La forme verbale au relatif ne peut apparaitre comme tête d'un groupe coordinatif ou unique forme verbale d'une phrase que lorsqu'elle a une valeur modale (cf. ci-dessous II.2.2).

II.1.2. L'aspect. La catégorie morphologique verbale bafia d'aspect caractérise l'événement décrit par le verbe soit comme dépourvu d'étendue temporelle, ou à l'opposé comme se déroulant pendant un certain temps, soit comme un comportement habituel, ou à l'opposé comme la répétition dans une période déterminée dudit événement. Il joue donc un rôle sémantique important.

L'aspect en bafia comporte quatre grammèmes :

a) Le ponctuel [PONC]. L'aspect ponctuel caractérise l'événement présenté comme un point dépourvu de toute étendue temporelle. Son interprétation varie en fonction du temps auquel il est manifesté. Au présent, il caracté- 
rise l'événement comme achevé, sans pour autant fixer l'attention sur l'achèvement comme tel, comme on pourrait le croire.

(10) Àrógà à-kàn-í 3,I-PRES,ABS-écrire-PONC

káàtà. Aroga «vient d'écrire» une lettre.

b) Le duratif [DUR]. L'aspect duratif caractérise l'événement comme se déployant de façon continue dans une étendue temporelle déterminée, c'est-àdire au moment de son accomplissement.

(11) Àrógà á-kànkàn 3,I-PRES,ABS-écrire,DUR Aroga est en train d'écrire une lettre.

c) L'habituel $[\mathrm{HAB}]$. L'aspect habituel caractérise l'événement comme ayant lieu d'habitude, sans localisation temporelle précise.

(12) Àrógà á-kàn-gà? 3,I-PRES, ABS-écrire-HAB

Aroga écrit souvent des lettres.

d) L'itératif [ITER]. L'aspect itératif caractérise l'événement comme répété à l'intérieur d'une période déterminée. L'événement en question doit avoir un début et une fin. Le nombre de répétitions est imprécis.
(13) Arógà á-kàngãokāngà? 3,I-PRES,ABS-écrire, ITER
Aroga écrit plusieurs fois des lettres.
boèkáàtà.

Les quatre aspects bafias ci-dessus se regroupent en deux sous-classes d'opposition : 1 . l'actuel : il regroupe le ponctuel et le duratif; 2 . le non-actuel : il regroupe l'habituel et l'itératif.

II.1.3. Le temps. La catégorie morphologique verbale bafia du temps situe l'événement sur l'axe du temps réel, par rapport au moment du discours. Elle comprend plusieurs grammèmes dont nous ne retenons que les deux qui nous intéressent : le présent et le quasi-passé.

II.1.3.1. Le présent [PRÉS]. Le présent bafia caractérise la réalisation de l'événement décrit par le verbe comme coïncidant plus ou moins avec le moment du discours. Tous les exemples cités jusqu'ici comportent une forme verbale au présent.

II.1.3.2. Le quasi-passé [QUAS.PASS]. Le quasi-passé bafia caractérise la réalisation d'un procès étendu dans la durée comme précédant de très peu (quelques heures au maximum) le moment du discours, et comme achevé.

Aroga vient d'échanger quelques propos avec son ami Zock, qui le quitte peu après. Absente de la scène immédiate, l'épouse d'Aroga a cru reconnaître la voix de Zock. Après le départ de ce dernier, elle tient à s'assurer de son impression. Elle pose alors la question suivante à son mari :
(14) Zò̀ à-tò-tóo
Zock parlait-il ici (tout à l'heure)? foé?
foé?
3,I-PRÉS,ABS-parler-QUAS-PASS

\section{II.2. Liste des valeurs sémantiques des grammèmes identifiés}

Le découpage catégoriel de la réalité qu'exprime le présent français est déjà beaucoup plus fin en bafia. En d'autres termes, l'aire sémantique couverte 
par le présent français connaît d'autres subdivisions du point de vue catégoriel en bafia. De ce point de vue, le bafia se révèle plus précis, et on peut prévoir que les grammèmes de cette langue seront moins polysémiques, puisqu'ils constituent déjà en eux-mêmes le résultat d'un effort de distinction sémantique plus poussé. Le phénomène est encore plus accentué pour les grammèmes de l'absolutivité qui ne sont justifiés quasi-exclusivement que par la syntaxe.

II.2.1. L'absolutif. Il n'a aucune valeur sémantique. Il est exclusivement utilisé à des fins syntaxiques, comme nous l'avons déjà souligné.

II.2.2. Le relatif. Il compte deux types de valeurs sémantiques, l'un lié à sa fonction dans le groupe verbal coordinatif, l'autre se rapportant au mode.

Dans le groupe coordinatif la valeur sémantique de la forme verbale bafia au relatif, tout comme pour son interprétation temporelle (cf.I.1.1.b), correspond à celle du verbe de la tête du groupe coordinatif en question. De ce point de vue, le relatif n'a donc pas de valeur sémantique propre.

Le relatif modal [RELB(MOD)]. Il apparait lorsque l'événement décrit par le verbe qui le manifeste est présenté comme une recommandation, une mise en garde, ou même un ordre. Il se rapproche donc de l'impératif qu'il remplace quand la réalisation de l'obligation qu'implique celui-ci relève du temps à venir. Il est par ce fait exclu de nos préoccupations présentes.

Il convient néanmoins de noter qu'ainsi employé, le relatif n'est plus assujetti aux contraintes syntaxiques indiquées plus haut (cf. II.1.1.b). Le verbe qui le manifeste peut donc être la tête d'une construction coordinative, en constituer un des autres éléments, ou être l'unique verbe de la phrase.

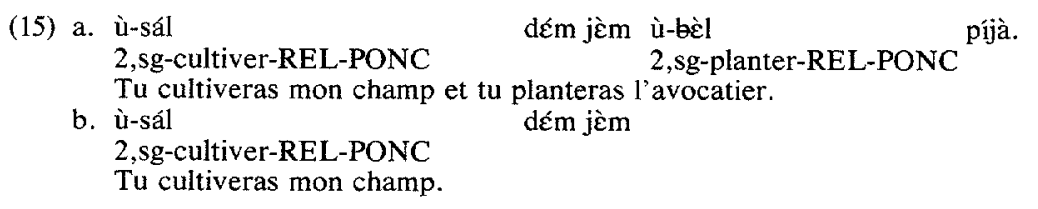

II.2.3. Le ponctuel. Il n'a qu'une valeur sémantique, manifestée lorsque la forme verbale en cause décrit un événement advenu une fois, et terminé depuis peu (quelques minutes). Ainsi, soit la phrase suivante :

(16) ì-kàn-í boèkáàtà.

1,sg-PRES-ABS-écrire-PONC

Je «viens d'écrire» des lettres.

Cette phrase ne peut être énoncée que si l'acte d'écrire est perçu comme un tout, comme accompli une fois, et comme terminé depuis peu.

II.2.4. Le duratif. Il n'a qu'une valeur sémantique, manifestée lorsque la forme verbale en cause décrit un événement dans son déroulement sur l'axe du temps. Il rappelle la forme progressive de la conjugaison anglaise.

(17) ń-kànkàn bòekáàtà
1,sg-PRES,ABS-écrire-DUR
Je suis en train d'écrire des lettres

II.2.5. L'habituel. Il n'a qu'une valeur sémantique, manifestée lorsque la forme verbale en cause décrit un événement qui advient souvent. 
(18) ń-kàn-gà? 1,sg-PRES,ABS-écrire-HAB

J'écris souvent des lettres.

boèkáàtà.

II.2.6. L'itératif. Il n'a qu'une valeur sémantique, manifestée lorsque la forme verbale en cause décrit un événement qui advient à plusieurs reprises et qui n'est pas réel au moment du discours.

(19) ń-kànā?kāngà?

1,sg-PRES,ABS-écrire,ITER

boèkáàtà.

J'écris des lettres à plusieurs reprises.

II.2.7. Le présent. Le présent bafia a trois valeurs sémantiques intrinsèques et une valeur contingente.

a) Valeurs sémantiques intrinsèques du présent bafia

1. Le présent réel [PRES $\mathrm{B}(\mathrm{REEL})]$. Il apparaît lorsque la forme verbale décrit un événement dont la réalisation coïncide plus ou moins avec le temps du discours.

2. Le présent historique [PRES B(HIST)]. Son apparition est assujettie en gros aux mêmes conditions que celles du présent historique français (cf. I.2.2).

(20) Báàbàn à-díi 3,I-PRES, ABS-manger-PONC Baban goûte (mange) de l'avocat quand il est chef.

píjà jèc à-báa

3,I-PRES,ABS-être-PONC

rífòm

3. Le présent gnomique [PRESB(GNOM)]. Son apparition est également assujettie à des conditions similaires à celles du présent gnomique français (cf. II.2.3). La différence consiste en son absence dans les proverbes, qui tient à la génèse de ces derniers en bafia: ils résument généralement une histoire vraie ou supposée telle, ce qui les rejette dans le passé, les excluant par là du domaine intemporel, caractéristique du présent gnomique des proverbes européens.
(21) Bée ìnoé ì-kpán-f́ á boèkpà? 3,IX-PRES,ABS-arriver-PONC
Ce chemin mène à Bafia.

b) Valeur sémantique contingente du présent bafia

Le présent bafia peut remplacer le futur lorsque la forme verbale décrit un événement dont la réalisation est supposée advenir dans un avenir assez proche. L'interprétation future d'un tel présent est souvent déduite du contexte de l'énoncé.

Aroga rend visite à Zock son ami et le trouve en train de faire ses valises pour un prochain voyage à Paris. Ils échangent quelques propos concernant ce voyage tant désiré et jamais réalisé jusqu'à ce moment. Alors Aroga, d'un air envieux lance le commentaire suivant :

(22) a. ù-kés

2,sg-PRES,ABS-aller-PONC

Tu vas à Paris cette fois-ci!

b. ú-pèsénpésèn

2,sg-PRES, ABS-quitter,DUR

Quand pars-tu d'ici?

á Pèrèsf́ čáač̀̆ !

fín?

II.2.8. Le quasi-passé. Il comporte deux valeurs sémantiques, l'une réelle, l'autre historique. 
a) Le quasi-passé réel. Il apparait lorsque la forme verbale en cause décrit un événement dont la réalisation relève d'un passé très récent de quelques heures au maximum (cf. exemple (14)).

b) Le quasi-passé historique. Il apparaît dans des conditions quasisimilaires à celles du présent historique français ou bafia, la seule différence étant que dans le cas du quasi-passé historique, la forme verbale doit décrire un événement dont la réalisation comporte une certaine durée sur l'axe temporel.

\section{II.3. Combinatoire des valeurs sémantiques des grammèmes bafias}

Ainsi qu'il a déjà été souligné dans l'introduction, toute forme verbale bafia à l'indicatif comporte obligatoirement des marques de l'absolutivité, de l'aspect et du temps. Il a également été relevé que les valeurs sémantiques des grammèmes de ces catégories sont manifestées selon une combinatoire bien précise. Le tableau 3 résume les combinaisons de valeurs possibles. Il comporte deux entrées: en abcisse les deux temps, subdivisés chacun en ses valeurs, en ordonnée, les aspects. Un plus $\left(_{+}\right)$représente une combinaison autorisée, un moins (_) une combinaison non autorisée.

\section{TABLEAU 3}

Combinatoire des valeurs sémantiques des grammèmes bafias.

\begin{tabular}{lcccccc}
\hline & \multicolumn{2}{c}{ tempset } \\
& valeurs & \multicolumn{2}{c}{} & \multicolumn{2}{c}{ quasi-passé } \\
\hline aspects & réel & historique & gnomique & contingent & réel & historique \\
ponctuel & + & + & + & + & - & - \\
duratif & + & - & - & + & + & + \\
habituel & + & - & + & - & - & + \\
itératif & + & - & + & - & - & + \\
\hline
\end{tabular}

Le' présent historique ne se combine pas avec le duratif, l'habituel et l'itératif parce que le premier requiert que la réalisation de l'événement décrit par le verbe soit obligatoirement limitée au temps révolu, alors que les aspects cités ne la limitent pas de la même façon.

Le présent gnomique et le duratif s'excluent parce que le premier marque le temps du verbe comme non-circonscrit alors que le second le marque comme fortement circonscrit au moment de la parole.

Le quasi-passé historique ne se combine pas avec le ponctuel par le fait que le présent historique au ponctuel joue déjà le rôle sémantique qui autrement serait échu au quasi-passé ponctuel. On sait d'ailleurs qu'en réalité le ponctuel au présent décrit l'événement comme accompli, donc en quelque sorte situé dans un passé très récent (il y a quelques minutes).

III - COMMENTAIRE DES DIFFICULTÉS DE CORRESPONDANCE

L'étude des valeurs sémantiques du présent français d'une part, et des grammèmes de l'absolutivité et l'aspect, ainsi que du présent et du quasi-passé 
bafia de l'autre, révèle une certaine similitude entre les significations encodées par le verbe dans les deux langues. Cela contribue à réduire les différences observables à ce propos entre elles, à la surface morphologique. Ces différences se situent d'ailleurs à deux niveaux :

1 - Niveau des valeurs sémantiques : le français distingue quatre valeurs intrinsèques du présent alors que le bafia n'en distingue que trois. Mais cette lacune est compensée au plan aspectuel dont le bafia tire ses ressources pour exprimer le même sens qu'en français, le faisant avec plus de détails. Ainsi, le français et le bafia comportent tous deux un historique et un gnomique du présent (plus discret en bafia où trois aspects sont distingués). Mais l'actuel et l'habituel du présent français sont exprimés en bafia par des distinctions aspectuelles, soit le duratif d'une part et l'habituel ou l'itératif de l'autre. Il convient de mentionner que le bafia tire avantage de la valeur historique du quasi-passé pour enrichir ses possibilités d'expression en ce qui concerne la valeur historique comme telle.

Pour ce qui est des valeurs contingentes du présent, elles sont plus nombreuses en français (elles y touchent soit le passé récent, soit le futur plus ou moins éloigné) qu'en bafia (seul le futur est concerné). Mais cette différence apparente est résorbée par la diversité des moyens d'expressions du bafia. En effet, lorsqu'il s'agit d'une forme verbale décrivant un événement dont la réalisation est reléguée au passé récent en français, le bafia utilise soit le présent ponctuel, la nuance aspectuelle étant respectée, soit le quasi-passé réel, dans les limites des significations en cause. Lorsque la forme verbale française décrit un événement de réalisation future, le bafia utilise soit le présent ponctuel, soit le présent duratif tel qu'indiqué auparavant (II.2.7.6).

2 - Niveau morphologique : alors que le français évite surtout de rendre obligatoire le choix des valeurs aspectuelles et de la place des verbes dans une structure coordinative, le bafia adopte plutôt cette organisation des significations. La comparaison de la situation dans les deux langues permet d'ailleurs de mettre en lumière une structure, latente dans les descriptions consultées des significations en cause, des valeurs aspectuelles du français. Il serait d'ailleurs intéressant d'élargir l'étude pour voir si ladite structure couvre les autres temps verbaux du français.

\section{IV - RÈGLES DE CORRESPONDANCES SÉMANTIQUES}

La forme générale des règles ci-dessous est la suivante:

$$
\mathrm{X} \Leftrightarrow \mathrm{Y} \mathrm{C}
$$

où $\mathrm{X}$ est l'entrée d'une règle donnée, une des valeurs du présent français; $\mathrm{Y}$ est la sortie de la règle en cause, constituée par les valeurs des grammèmes des catégories correspondantes du bafia; $\Leftrightarrow$ indique la correspondance; et $\mathrm{C}$ est l'ensemble des conditions sémantiques ou syntaxiques de la correspondance en question.

Le verbe décrit toujours un événement (état ou action). 


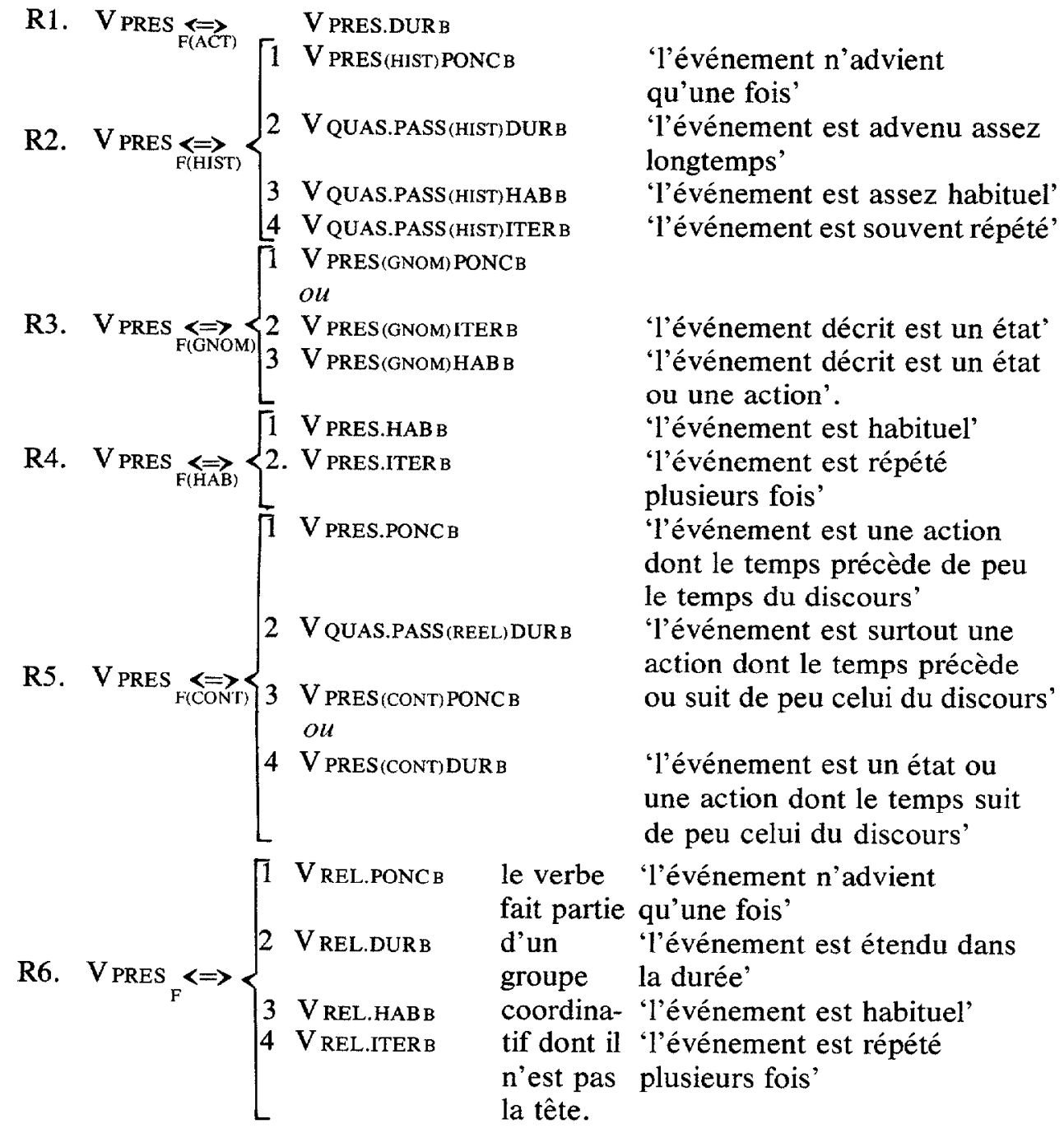

Exemples

Chacun des exemples ci-dessous illustre une règle ou une sous-règle. La lettre $\mathrm{R}$ indique une règle quelconque; le premier chiffre en indice indique le numéro de la règle en question, le second, le numéro de la sous-règle correspondante.

R1. Aroga écrit une lettre maintenant Àrógà á-kànkàn 3,I-PRES,ABS-écrire,DUR

R2-1. Baban écrit cette lettre étant chef Báàbàn à-kàn-́ 3,I-PRES,ABS-écrire-PONC

R2-2. Baban écrit des lettres étant chef Báàbàn à-kànkán-́́ 3,I-écrire-QUAS.PASS-DUR káàta čaamèn kf.

káàtà ànoé dí à-rì 3,I-PRES,ABS-être-PONC rìfòm. bcèkáàtà jèć à-báa 3,I-être-QUAS-PASS-DUR rùfòm. 
R2-3. Baban «prend l'habitude» d'écrire des lettres étant chef

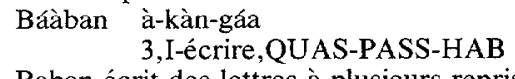

R2-4. Baban écrit des lettres à plusieurs reprises étant chef.

Báàban à-kàngáakàngà ? bœekáàta jèc àbáa ràfòm.

3,I-écrire,QUAS-PASS-ITER

R3-1. Ce chemin mène à la ville.

$$
\text { Bée ìnó ì-kpáy-i } 3 \text { b́ bàn. }
$$

R3.2 Ce chemin mène généralement à la ville.

Bée inò jéc̀-kpāgāPkpāgà?

bí bàn.

3,IX-PRES, ABS-arriver,ITER

R4-1. La terre tourne autour du soleil.

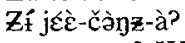

jwós

3,IX-PRES, ABS-tourner, faire-HAB

R4-2. Aroga écrit d'habitude des lettres.
Àróga
á-kàn-gà?
boèkáà̀à.

3,I-PRES,ABS-écrire-HAB

R5-1. Aroga vient de descendre de la voiture ça ne fait pas longtemps.

Ârógà à-sén-í moètúwà kí-ká-fínén-bí.
3,I-PRES,ABS-descendre-PONC
3,VII-PRES, ABS-pas-durer-PONC-encore

R5-2. Aroga descendait de voiture ça ne fait pas longtemps.

$\begin{array}{ll}\text { Àrógà } & \text { à-sénsén-́́ } \\ & 3, I-d e s c e n d r e, Q U A S-P A S S, D U R\end{array}$

R5-3. Aroga amorce la construction de sa maison demain.

$\begin{array}{ll}\text { Àrógà à-túm- } \varepsilon \varepsilon & k \grave{x} \\ & 3, \mathrm{I}-\mathrm{PRES}, \mathrm{ABS}-\mathrm{commencer-PONC}\end{array}$

4. Àrógà á-túméntúmèn

3,I-PRES,ABS-commencer,DUR

kìtón kf́ mée míi rìdúrí.

6-1. Aroga éternue et tousse à ce moment-ci.

Àrógà à-č́p?m-źc à-kósì

kìtón ká mée míi rìdúrí.

en train d'éternuer et de tousser.

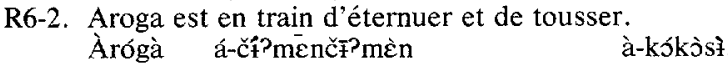

3,I-PRES,ABS-éternuer,DUR 3,I-REL-tousser,DUR

R6-3. Aroga éternue et tousse d'habitude (en parlant à un médecin d'un cas quasi chronique).

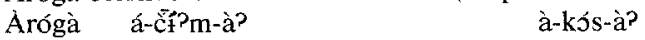

3,I-PRES,ABS-éternuer-HAB 3,I-REL-tousser-HAB

R6-4. Aroga éternue et tousse à plusieurs reprises

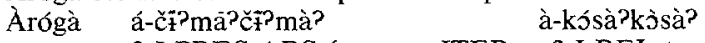

3,I-PRES, ABS-éternuer,ITER 3,I-REL-tousser,ITER

\section{BIBLIOGRAPHIE}

AROGA, D.P. et A. MARCHAL (1981): «Les phonèmes du bafia: Application des techniques de la phonétique expérimentale à l'étude des voyelles».

AROGA, D. P. et I.A. Mel'čuk (1981) : «Un modèle de la conjugaison verbale bafia à l'indicatif " (à paraître).

CHEVALIER, J.-C. et al. (1964) : Grammaire Larousse du français contemporain, Paris, Larousse. GREVISSE, M. (1980) : le Bon usage, Gembloux, Duculot.

MARTINET, A. et al. (1979) : Grammaire fonctionnelle du français, Paris, Didier.

MEL'CUK, I. A. (1974) : «Esquisse d'un modèle linguistique du type «Sens Texte», dans Problèmes actuels en psycholinguistique, Colloques internationaux du CNRS, $\mathrm{n}^{\circ}$ 206, Paris, p. 291-317.

MEL'CUK, I.A. (1978): "Théorie de langage, théorie de traduction», dans Meta, vol. 23, $n^{\circ} 4$.

SCHOGT, H.G. (1968) : le Système verbal du français contemporain, The Hague, Mouton.

WAGNER, R. L. et al. (1962) : Grammaire du français classique et moderne, Paris, Hachette. 